\title{
DURABILIDADE NATURAL DA MADEIRA DE PUPUNHA (Bactris gasipaes KUNTH). I. FUNGOS.
}

\author{
Maria Aparecida de JESUS*, Raimunda Liege Souza de ABREU*
}

RESUMO - A durabilidade da madeira de Bactris gasipaes Kunth (pupunha) face aos fungos foi avaliada em ambiente florestal e urbano e em condições de laboratório. Foram utilizadas, cinco árvores com espinhos e cinco sem espinhos da população Tabatinga, raças Putumayo, sendo retirados discos de $25-30 \mathrm{~cm}$ da base, meio e do topo de cada pupunheira e colocados em uma área florestal próxima ao Banco Ativo de Germoplama de B. gasipaes ( $\left.2^{\circ} 38^{\circ} \mathrm{S}, 60^{\circ} 03^{\prime} \mathrm{W}\right)$. Os discos foram inspecionados trimestralmente, para acompanhar o processo de biodeterioração da madeira causado por fungos durante dezoito meses. No ensaio em ambiente urbano, as amostras do estipe foram distribuídas, uma na posição côncava e outra na convexa, sobre uma estrutura de madeira localizada em uma área próxima da CPPF do Campus do INPA, Manaus e inspecionadas bimestralmente por um ano. Em ambiente florestal, o revestimento dos discos mostrou-se suscetivel aos fungos Hymenogramme javanensis Mont. \& Berk. e Hypoxylon rubiginosum (Pers.: Fr.) Fr.. Em ambiente urbano, a madeira apresentou alta susceptibilidade a Lenzites striata (Swart.: Fr.) Fr. e a Lenzites sp. e o revestimento a Porostereum amethysteum Hjortst. \& Ryv. e a Stereum strigoso-zonatum (Sch.) G. H. Cunn.. Em condiçōes de laboratório, a madeira da pupunheira demonstrou alta resistência aos fungos Lenzites trabea Pers.:Fr., Polyporus fumosus Pers.:Fr. e Pycnoporus sanguineus (L.:Fr.) Murr.

Palavras-chave: Bactris gasipaes Kunth, biodegradaçāo, fungos xilófagos, durabilidade natural.

\section{Natural Durability of Peach Palm (Bactris gasipaes Kunth) Wood. I. Fungi}

ABSTRACT - The durability of the stem of Bactris gasipaes Kunth (Peach palm) was evalu ated in field tests in a forest and in an urban environment, as well as in laboratory conditions. Five trees with spines and five trees without spines from the Tabatinga population (Putumayo landrace) were used in the experiment. Disks approximately $25-30 \mathrm{~cm}$ thick were taken from the butt, middle and top portions of each tree and distributed at random in forest area near the INPA Peach Palm Germoplasm Bank ( $\left.2^{\circ} 38^{\prime} S 60^{\circ} 03^{\prime} \mathrm{W}\right)$. The disks were checked every three months for 18 months for susceptibility to fungi. In the urban environment each disk was halved to remove the center and each half was distributed at random, one in a concave and the other in a convex position, on a wooden framework in an area near the CPPF/INPA, Manaus, and checked every 2 months for one year. In the forest environment the coating of the disks was susceptible to the fungi Hymenogramme javanensis Mont. \& Berk. and Hypoxylon rubiginosum (Pers.: Fr.) Fr. In the urban environment the wood was highly susceptible to the fungi Lenzites striata (Swart.: Fr.) Fr., Lenzites sp, Porostereum amethysteum Hjortst.\& Ryv. and Stereum strigoso-zonatum (Sch.) G. H. Cunn. In laboratory conditions the peach palm wood showed great resistance to the fungi Lenzites trabea Pers.: Fr., Polyporus fumosus Pers.: Fr. and Pycnoporus sanguineus (L.: Fr.) Murr.

Key-words: Bactris gasipaes Kunth, biodeterioration, fungi decay, natural durability.

\section{Introdução}

Bactris gasipaes Kunth (pupunha) é uma palmeira tropical que apresenta potencial de uso de todas as suas partes na agroindústria, principalmente a extração do palmito. A palmeira apresenta crescimento rápido, tornando-se alta demais para coletar os frutos com facilidade. Neste caso, é indicada a renovação do plantio, eliminando-se alguns dos

* CPPF/Instituto Nacional de Pesquisas da Amazônia, Av. André Araújo, 2936, Caixa Postal 478, 69011-970, Manaus, AM, Brasil. E-mail: ranna@inpa.gov.br 
estipes (Araújo, 1991; Gomes, 1993).

Para fins práticos, como as palmeiras são árvores monocotiledôneas muitos especiais por não serem lenhosas, (Marchiori, 1995), os elementos estruturais do estipe da pupunheira foram considerados nos seguintes termos: revestimento, medula (medula central formada por numerosos feixes vasculares do tecido condutor) e madeira (tecido vascular primário), a parte entre a medula e o revestimento (Lorenzo et al., 1996).

A madeira do estipe da pupunheira é de cor parda-escura, com fibras amarelas, forte, durável, fácil de trabalhar, aceitando um bom polimento (Mora Urpí, 1984). Tais características favorecem a utilização da madeira na confecção de utensílios usados pelos Ameríndios. Alguns destes utensílios foram adaptados pelas indústrias de artesanato na Colômbia (Patiño, 1963). aproveitamento do estipe vem sendo estudado com fim de uso na construção habitacional, na confecção de pequenos objetos de madeira, instrumentos musicais e móveis (Lobato et. al., 1999.)

Dentre as propriedades da madeira, a durabilidade natural é um aspecto importante, pois fornece dados da resistência ou da susceptibilidade da madeira aos organismos xilófagos. A avaliação desta propriedade é feita por meio de ensaios em condições de laboratório e de campo. De modo geral, estes ensaios têm recebido considerável aceitação no mercado madeireiro, por gerar informações sobre as diferentes classes de resistência da madeira, além dos cuidados a serem adotados com respeito à aplicabilidade da madeira em ambiente interno e externo.

Neste contexto, o presente trabalhso teve como objetivo avaliar a durabilidade natural da madeira de $B$. gasipaes (pupunha) em condições de laboratório, ambiente florestal e urbano, como também verificar a ocorrência de fungos xilófagos no estipe da pupunheira exposta nestes dois ambientes.

\section{Material e Métodos Características da Área do Banco Ativo de Germoplasma de $B$. gasipaes (Pupunha)}

O Banco Ativo de Germoplasma de $B$. gasipaes está localizado na rodovia Manaus-Boa Vista, Km 40 $\left(2^{\circ} 38^{\prime} \mathrm{S}, 60^{\circ} 03^{\prime} \mathrm{W}\right)$. O solo é do tipo latossolo amarelo, com textura muito pesada em todo o perfil e integrada por solos profundos, bastante desgastados, fortemente ácidos e bem drenados, com teor de argila acima de $70 \%$ no horizonte B (Falesi, 1971). O clima da área, segundo classificação de Köppen, é do tipo Ami "A" (clima tropical chuvoso), onde a temperatura média anual é $26,7^{\circ} \mathrm{C}$, com valores médios para as máximas e mínimas de $31,2^{\circ} \mathrm{C}$ e $23,5^{\circ} \mathrm{C}$, respectivamente. As chuvas apresentam índice pluviométrico anual na ordem de $2101 \mathrm{~mm}$, distribuídos em duas épocas distintas, isto é, uma época chuvosa e uma mais seca. A época chuvosa ocorre entre os meses de novembro e maio, sendo o mês de março o que apresenta maior índice 
pluviométrico e a outra se estende de junho a outubro. A umidade relativa apresenta média anual de $84 \%$, variando entre $77 \%$ a $88 \%$ (Vieira \& Santos, 1987).

\section{Durabilidade natural da madeira de $B$. gasipaes em ambiente florestal}

Como as palmeiras são constituídas de estipe (tronco), tornou-se necessária à definição da terminologia dos elementos estruturais (revestimento, medula e madeira) das partes ensaiadas. Foram selecionadas cinco pupunheiras com espinhos e cinco sem espinhos da população Tabatinga (raça Putumayo), apresentando altura média entre 15 e $20 \mathrm{~m}$ e diâmetro médio de 25 a $30 \mathrm{~cm}$, do Banco Ativo de Germoplasma de Pupunha. De cada árvore foram cortados discos de $30 \mathrm{~cm}$ de espessura, secionados da base, meio e topo do estipe, sendo que cada disco foi codificado com uma placa contendo o número, a posição, a procedência e a variedade da pupunheira.

O ensaio de durabilidade natural dos discos de pupunheira foi instalado em uma área próxima ao plantio das pupunheiras, caracterizada como vegetação de capoeira. A área foi dividida em dois blocos e cada bloco continha seis fileiras com cinco discos em cada, distribuídos aleatoriamente sobre o solo e sob a vegetação, a uma distância de $50 \mathrm{~cm}$ uns dos outros. Os discos foram inspecionados trimestralmente entre outubro de
1995 e janeiro de 1997. O nível de biodegradação no revestimento, na madeira e na medula causado por fungos, foi avaliado de acordo com os critérios relacionados na Tabela 1 , descritos por Serpa (1982). Durante as inspeções, cada disco era removido e submetido a uma pequena pressão em sua superfície com um instrumento pontiagudo (formão) para se determinar um eventual amolecimento (deterioração) ou quebra. Caso o disco não estivesse totalmente deteriorado, o mesmo era colocado novamente na posição em contato com o solo. A durabilidade natural foi determinada quando $60 \%$ dos discos apresentaram o índice de comportamento igual a zero e também com base na vida útil (Tab. 2) proposto por Cavalcante et al. (1982). Os exemplares dos fungos que estavam colonizando os discos foram coletados.

\section{Ensaio em ambiente urbano}

Na montagem do ensaio, adotouse o mesmo procedimento usado na amostragem da pupunheira exposta ao ambiente florestal. Como se pretendia a utilização da madeira em ambiente externo e interno e também como telha, assoalho, dentre outros, o disco foi secionado axialmente para a retirada da medula, parte não lignificada. Cada metade foi codificada com uma placa contendo o número, a posição, a procedência e a variedade da pupunheira. Posteriormente, as amostras foram distribuídas aleatoriamente, uma na posição convexa e a outra na côncava, 
sobre três estruturas de madeira medindo cada uma $70 \mathrm{~cm}$ de altura, $20 \mathrm{~cm}$ de largura e $2 \mathrm{~m}$ de comprimento. $\mathrm{O}$ conjunto foi alocado em uma área com incidência homogênea dos raios solares, próxima da serraria da Coordenação de Pesquisas em Produtos Florestais do Instituto Nacional de Pesquisas da Amazônia em Manaus.

\section{As}

amostras

foram inspecionadas bimestralmente, no período de setembro de 1996 a agosto de 1997 . O critério de avaliação da biodegradação foi o mesmo adotado no ensaio em ambiente florestal, como também os exemplares dos fungos que estavam colonizando as amostras foram coletados. Estes fungos, assim como os coletados no ambiente florestal, foram identificados por especialistas e depositados no Herbário do Instituto Nacional de Pesquisas da Amazônia, INPA.

\section{Durabilidade natural da madeira} de $B$. gasipaes em condições de laboratório

O método alternativo Agar-bloco para teste acelerado de laboratório, descrito por Sutter (1978), foi adaptado para a madeira da

Tabela 1. Classificaçầo do nível de deterioração das madeiras em ensaio de campo.

\begin{tabular}{|c|c|c|}
\hline Nota & $\begin{array}{c}\text { Indice de } \\
\text { Comportamento }(\%)\end{array}$ & Nivel de deterioração \\
\hline 0 & 100 & Ausência de sintoma de deterioraçäo \\
\hline 1 & 90 & $\begin{array}{l}\text { Deterioração superficial por fungos ou } \\
\text { térmitas }\end{array}$ \\
\hline 2 & 70 & $\begin{array}{l}\text { Deterioraçâo evidente, porém moderada } \\
\text { causada por fungos ou termitas }\end{array}$ \\
\hline 3 & 40 & $\begin{array}{l}\text { Deterioração intensa, ou colonização } \\
\text { intensa por fungos e térmitas }\end{array}$ \\
\hline 4 & 0 & $\begin{array}{l}\text { Quebra - perda quase total da } \\
\text { resistência }\end{array}$ \\
\hline
\end{tabular}

Fonte: Serpa (1982)

Tabela 2. Classificaçāo utilizada na avaliaçāo da durabilidade natural de madeira em contato com a solo

\begin{tabular}{lc}
\hline \multicolumn{1}{c}{ Classe de durabilidade } & Vida útil (em anos)* \\
\hline Altamente durável & $>8$ \\
Durável & de 5 a 8 \\
Moderadamente durável & de 2 a 5 \\
Nào durável & de 0 a 2 \\
\hline
\end{tabular}

Fonte: Cavalcante (1982) * Tempo decorrido para que $60 \%$ das amostras apresentem o comportamento $=0$ 
pupunheira. Os fungos Polyporus fumosus Pers.:Fr. e Pycnoporus sanguineus (L.:Fr.) Murr., causadores da podridão branca e Lenzites trabea Pers.:Fr., causador da podridão parda, foram usados no bioensaio.

As amostras foram retiradas da parte basal de três pupunheiras com espinho e três sem espinho, pois as demais alturas do estipe não apresentavam madeira suficiente para a confecção dos corpos de prova. Deste material foram confeccionadas 15 amostras da madeira, medindo 3,0 x $2,5 \times 0,6 \mathrm{~cm}$ (sendo a primeira medida na direção das fibras) para $o$ teste. Como testemunha, foram utilizadas cinco amostras de Simaruba amara Aubl. (marupá), medindo as mesmas dimensões das amostras-teste. Todas as amostras foram lixadas de modo a evitar futura desfibrilização e secas em estufa à $60^{\circ} \mathrm{C}$, com ventilação moderada por 24 horas. Posteriormente, foram mantidas em estufa com temperatura controlada à $28^{\circ} \mathrm{C}$, com ventilação moderada e pesadas semanalmente até a obtenção de peso constante (P1). Após esta etapa, as amostras foram autoclavadas por 15 minutos à $120^{\circ} \mathrm{C}$.

Primeiramente, para desenvolver a atividade lignocelulolítica dos fungos, usou-se amostras de marupá (alimentador) com as dimensões 10,3 x 0,9 x $0,6 \mathrm{~cm}$ (primeira dimensão paralela às fibras), também autoclavados por 15 minutos. No bioensaio, três alimentadores foram colocados paralelamente no meio de cultura Agar-malte, ainda semisólido. Após a solidificação do meio, distribuiu-se inóculos do fungo próximos aos alimentadores. As culturas foram acondicionadas em estufa com temperatura à $28^{\circ} \mathrm{C}$ e umidade relativa de $70 \%$, até o desenvolvimento dos fungos sobre os alimentadores.

$\mathrm{Na}$ montagem do bioensaio, distribuíram-se as cinco amostras de cada pupunheira sobre os alimentadores cobertos pelo fungo na placa de Petri. O mesmo procedimento foi adotado para as cinco amostras da testemunha. A cultura (fungo/amostra) foi mantida em estufa à $28^{\circ} \mathrm{C}$ por 16 semanas, e após este período, as madeiras foram limpas e colocadas em estufa à $60^{\circ} \mathrm{C}$ com ventilação moderada por 24 horas, sendo pesadas e mantidas nas mesmas condições até atingirem o peso constante (P2).

A avaliação da perda de massa foi calculada pela diferença do peso inicial (P1) com o peso final das amostras expostas aos fungos (P2), expressa em porcentagem e, com base nos valores da perda de massa, estabeleceu-se a classe de resistência natural da madeira aos fungos degradadores, relacionada na Tabela 3 , proposta pela American Society for Testing and Materials (1981).

\section{Resultados e Discussão Durabilidade natural da madeira de $B$. gasipaes em ambiente florestal \\ Os fungos apodrecedores de madeira iniciaram o processo de colonização mais intensamente na medula e no revestimento das alturas do meio e do topo a partir do terceiro}


Tabela 3. Classe de resistência da madeira ao ataque de fungos degradadores.

\begin{tabular}{cc}
\hline Média de perda de massa \% & Classificaçäo \\
\hline $0-10$ & Altamente resistente \\
$11-24$ & Resistente \\
$25-44$ & Resisténcia moderada \\
Rcirra oie 45 & Não resisterte \\
\hline
\end{tabular}

Fonte: (ASTM, 1981)

mês, sendo que o nivel de infestação mais elevado (nota 4) foi observado a partir do sexto mês e perdurou até o décimo quinto, principalmente na medula, enquanto que na madeira, eles frutificaram-se a partir do sexto mês (Tab. 4). A medula dos discos foi bastante colonizada por Coprinus sp (Coprinaceae). Estes fungos são saprófitas e se desenvolvem em substratos com umidade acima de $50 \%$, onde obtêm alimento dos resíduos do processo de biodegradação (Ortós, 1975). A colonização dos fungos Aleurodiscus cerussatus (Bres.) Höhn. \& Litsch., Hymenogramme javanensis Mont. \& Berk.. Hypoxylon rubiginosum (Pers.: Fr.) Fr., foi observada principalmente no revestimento dos dis$c o s$, sendo que os dois últimos foram os principais responsáveis pela deterioração dessa parte.

Dentre as partes ensaiadas, a madeira e a medula da pupunheira demostraram maior resistência aos fungos, o revestimento da pupunheira com espinho apresentou ser mais susceptivel e a madeira da parte basal demonstrou resistência moderada aos fungos (Tabela 4), Deste modo, a madeira de $B$. gasipaes pode ser considerada não durável, com base nos dados obtidos e na classificação da durabilidade natural de madeira proposta por Calvacante et al. (1982).

\section{Durabilidade natural de $B$. gasipaes em ambiente urbano}

Os fungos apodrecedores de madeiras iniciaram a infestação (nota 1) no revestimento e na madeira das três alturas do estipe no período entre quatro e seis meses de exposição. A madeira e o revestimento das amostras retiradas do topo de ambas variedades da pupunha apresentaram o maior nỉvel de deterioração (nota 4), a partir do oitavo mês de exposiçăo, enquanto que poucas amostras da parte do meio do estipe sem espinho apresentaram esse nivel de deterioração, ocasionado pelo ataque dos fungos. Por outro lado, nota-se que as amostras retiradas da parte basal de ambas variedades da pupunheira apresentaram deterioração intensa (nota 3) provocada pelos fungos (Tab. 5).

De modo geral, os fungos frutificaram nas amostras dispostas em ambas posiçōes, sendo que a maioria desenvolveu-se no revestimento das amostras na posição convexa e na madeira na posição côncava (Tab. 6). Por exemplo, Lenzites striata (Swart.: Fr) Fr. e Lenzites sp frutificaram na madeira, enquanto que Porostereum amethysteum Hjortst. \& Ryv. e Stereum strigoso-zonatum (Sch.) G. H. Cunn, desenvolveram-se muito bem, 
Tabela 4. Nível de deterioração causado por fungos no revestimento, medula e madeira de discos retirados da base, meio e topo da pupunheira com e sem espinhos plotados em ambiente florestal durante 18 meses.

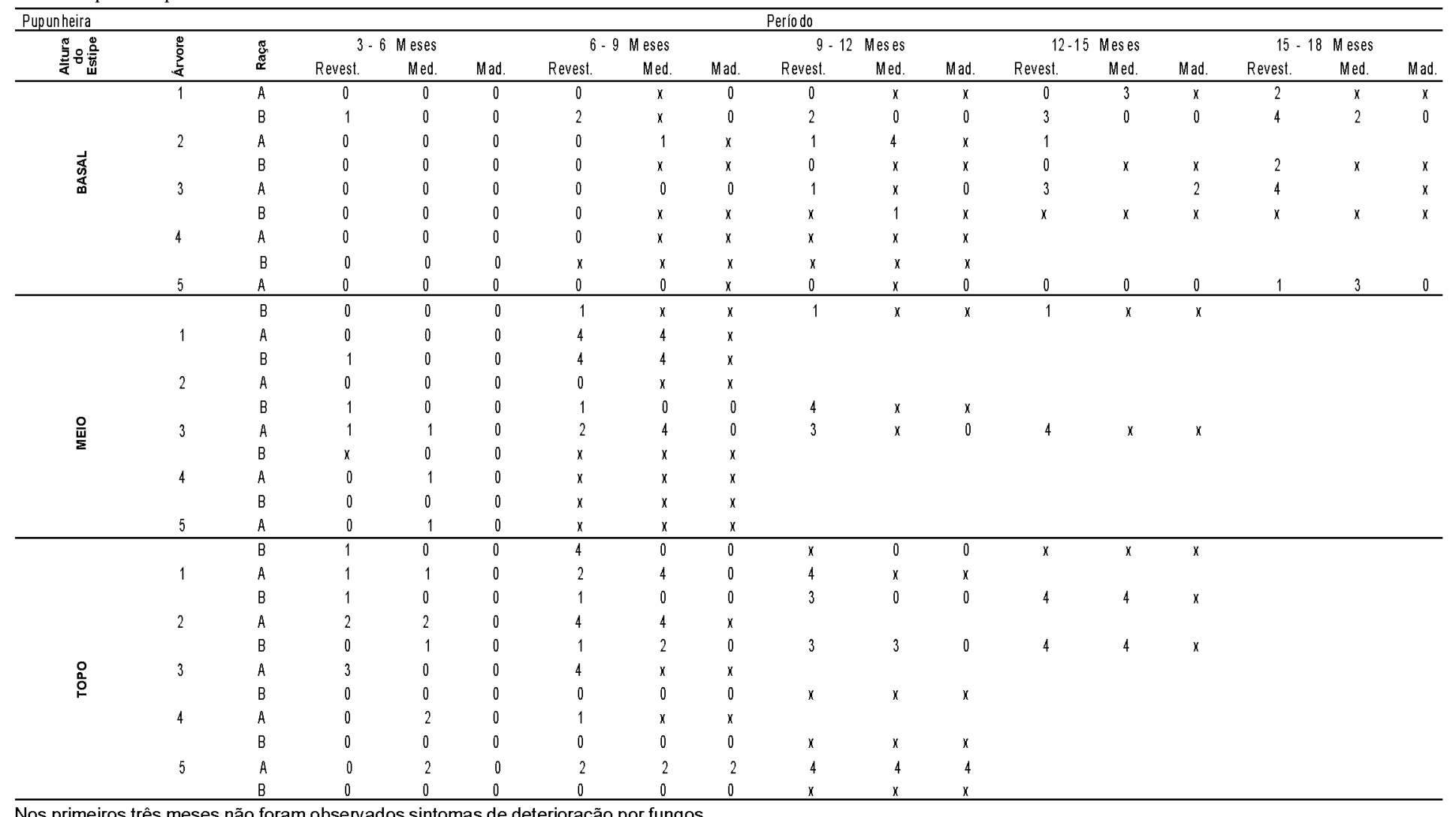


Tabela 5. Nível de deterioração causado por fungos no revestimento e madeira de discos retirados das alturas da base, meio e topo da pupunheira com e sem espinhos plotados em ambiente urbano durante 12 meses.

\begin{tabular}{|c|c|c|c|c|c|c|c|c|c|c|}
\hline \multirow{2}{*}{ 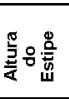 } & \multirow{2}{*}{$\frac{0}{3}$} & \multirow{2}{*}{ 啬 } & \multicolumn{2}{|c|}{$4 \cdot 6$ Meses } & \multicolumn{2}{|c|}{$6-8$ Meses } & \multicolumn{2}{|c|}{$8 \cdot 10$ Meses } & \multicolumn{2}{|c|}{$10 \cdot 12$ Meses } \\
\hline & & & Rev estestim ento & Madeira & Rev estimento & Madeira & Revestimento & Madeira & Revestimento & Madeira \\
\hline \multirow{9}{*}{$\begin{array}{l}\vec{d} \\
\text { 哥 } \\
\text { 品 }\end{array}$} & \multirow[t]{2}{*}{1} & A & 0 & 0 & 0 & 0 & 0 & $x$ & $x$ & $x$ \\
\hline & & $B$ & 0 & 0 & 0 & 0 & 2 & 2 & $x$ & $x$ \\
\hline & \multirow[t]{2}{*}{2} & A & 1 & 0 & 2 & $x$ & & & & \\
\hline & & $B$ & 0 & 0 & 0 & 0 & $x$ & 2 & 1 & $x$ \\
\hline & \multirow[t]{2}{*}{3} & A & 1 & 0 & 2 & 2 & 3 & 3 & $x$ & $x$ \\
\hline & & $B$ & 0 & 0 & 0 & 0 & $x$ & $x$ & & \\
\hline & \multirow[t]{2}{*}{4} & A & 1 & 0 & 0 & 0 & $x$ & 2 & $x$ & $x$ \\
\hline & & $B$ & 1 & 0 & 2 & 0 & 3 & 0 & 3 & 0 \\
\hline & \multirow[t]{2}{*}{5} & $A$ & 1 & 0 & 2 & 0 & 1 & $x$ & & \\
\hline \multirow{10}{*}{$\frac{\mathrm{O}}{\underline{\underline{z}}}$} & & $\mathrm{~B}$ & 1 & 0 & 2 & 0 & 1 & 2 & $x$ & $x$ \\
\hline & \multirow[t]{2}{*}{1} & A & 1 & $x$ & $x$ & 1 & $x$ & $x$ & & \\
\hline & & $B$ & 1 & $x$ & $x$ & 1 & & & & \\
\hline & \multirow[t]{2}{*}{2} & A & 1 & $x$ & 1 & 1 & 3 & 3 & $x$ & $x$ \\
\hline & & $B$ & 1 & $x$ & $x$ & 1 & $x$ & 2 & $x$ & $x$ \\
\hline & \multirow[t]{2}{*}{3} & $A$ & 1 & $x$ & $x$ & 2 & $x$ & $x$ & $x$ & $x$ \\
\hline & & B & 1 & $x$ & 1 & 2 & 4 & 4 & & \\
\hline & \multirow[t]{2}{*}{4} & $A$ & 1 & $x$ & $x$ & 2 & $x$ & $x$ & $x$ & $x$ \\
\hline & & $B$ & 1 & $x$ & $x$ & 2 & 3 & 3 & $x$ & $x$ \\
\hline & \multirow[t]{2}{*}{5} & A & 1 & $x$ & $x$ & 2 & & & & \\
\hline \multirow{11}{*}{$\begin{array}{l}\circ \\
\vdots \\
\circ\end{array}$} & & $B$ & 1 & $x$ & 2 & 2 & 4 & 4 & & \\
\hline & \multirow[t]{2}{*}{1} & $A$ & 1 & $x$ & 2 & 1 & 3 & 4 & 3 & $x$ \\
\hline & & $B$ & 1 & $x$ & 1 & 1 & 3 & 2 & 4 & 4 \\
\hline & \multirow[t]{2}{*}{2} & $A$ & 1 & $x$ & 2 & $x$ & 4 & 4 & & \\
\hline & & $B$ & 1 & $x$ & $x$ & $x$ & $x$ & $x$ & $x$ & $x$ \\
\hline & \multirow[t]{2}{*}{3} & $A$ & 1 & $x$ & $x$ & $x$ & $x$ & $x$ & $x$ & $x$ \\
\hline & & $B$ & 1 & $x$ & 3 & 3 & 4 & 4 & & \\
\hline & \multirow[t]{2}{*}{4} & $A$ & 1 & $x$ & 3 & 3 & 4 & 4 & & \\
\hline & & $B$ & 1 & $x$ & 3 & 3 & 4 & 4 & 4 & $x$ \\
\hline & \multirow[t]{2}{*}{5} & $A$ & 1 & 1 & 3 & 3 & 4 & 3 & $x$ & $x$ \\
\hline & & B & 1 & $x$ & 2 & $x$ & 2 & $x$ & 2 & $x$ \\
\hline
\end{tabular}

Nos primeiros quatro meses não foram observados sintomas de deterioração por fungos

Ausência de sintoma de deterioração; 1 = Deterioração superficial; 2 = Deterioração moderada Deterioração intensa; 4 = Quebra - perda quase total da resistência; $x$ = Deterioração por outros organismo xilófagos. 
praticamente em toda a extensão do revestimento. Observou-se que os carpóforos desenvolveram-se melhor nas amostras expostas na posição convexa, provavelmente pelo fato de que as amostras nesta posição ficam mais expostas à luz, fator importante para o crescimento das espécies (Ortós, 1975).

Os fungos Junghuhnia crustacea (Jungh.) Ryv., L. striata, Lenzites sp, $P$. amethysteum, Schizopora paradoxa (Fr.) Donk, Stereum strigoso-zonatum (Sch.) G.H. Cunn., Trametes cf. cotonea (Pat. \& Har.) Ryv., T. cf. pubescens (Schum.Fr.) Pil. e T. villosa (Fr.) Kreisel destacaram-se em número de ocorrência nas amostras. Também, as espécies saprófitas Nodulisporium gregorium (B. \& C.) Meyer Phaeiosoria sp e Rhytidhysteron rufulum (Spreng.) Petr. colonizaram principalmente as amostras do topo e do meio da pupunheira, concentrando seu ataque no revestimento e na posição convexa. Dentre estas espécies, Lenzites striata e Lenzites sp, encontradas na madeira e em ambas as posições, causam podridão parda em madeira exposta em ambiente externo (Overholts, 1977), enquanto que $J$. crustacea, $T$. cotonea e $T$. vilosa, encontradas no revestimento na posição convexa, são causadoras de podridão branca (Ryvarden \& Jonhanser, 1980). Panus crinitus (L.:Fr) Sing., P. cf. nicotianus Berk, e Pycnoporus sanguineus (L.: Fr.) Murr. foram as únicas espécies encontradas em todas as partes do disco, destacando na madeira as duas posições ensaiadas (Tab. 6). Estes fungos, juntamente com Schizophyllum commune Fr., $S$. fasciatus, T. cotonea, S. strigosozonattum T. cf. pubescens e T. villosa, também ocorrem em madeiras serradas ou trabalhadas expostas às intempéries (Calvacanti, 1983; Jesus, 1985; 1995; Milano et al., 1985).

Comparando a diversidade dos fungos na madeira da pupunheira exposta em ambiente florestal e urbano, verifica-se que a madeira apresenta suscetibilidade a poucas espécies de fungos no ambiente florestal, enquanto que a madeira demonstra ser altamente suscetível às várias espécies de fungos no ambiente urbano. A diversidade fúngica encontrada na madeira exposta ao ambiente urbano pode estar relacionada com a grande quantidade de resíduos lignocelulolíticos (toras, serragem, entre outros) estocados no pátio da serraria, substratos que representam uma potente fonte de inóculo de fungos. Por outro lado, o número reduzido de espécies nos discos expostos ao ambiente florestal pode estar associado com a alta umidade e pouca luminosidade e material lignocelulolítico. Esta diversidade fúngica é pequena comparada à encontrada em discos de diversas essências florestais (folhosas), expostos no mesmo ambiente florestal (INPA/CPPF, 1991; Jesus 1995).

Também, a composição de espécies nas amostras do estipe de $B$. gasipaes expostas no ambiente 
florestal e urbano difere podridão na madeira.

significativamente, considerando que Concomitante com as várias apenas a espécie $A$. cerussatus foi espécies de fungos, tanto no ambiente comum aos dois ambientes (Tab. 6). florestal como no urbano, ocorreu o Além disso, a maioria das espécies ataque de outros organismos xilófagos, encontradas no ambiente urbano são os quais ocasionaram diferentes níveis relatadas como causadoras de de deterioração, que serão avaliados

Tabela 6. Ocorrência de fungos no revestimento, medula e madeira expostos ao ambiente florestal e no revestimento e na madeira de discos de pupunheira expostos nas posições côncava e convexa em ambiente urbano

\begin{tabular}{|c|c|c|c|}
\hline Familias & Espécies & Ambiente florestal & Ambiente urbano \\
\hline Coprinaceae & Coprinus sp & ME & \\
\hline \multirow[t]{8}{*}{ Corticiaceae } & Aleurodiscus cerussatus (Bres.) Höhn. \& Litsch & RE & $\mathrm{RE}, \mathrm{CE}$ \\
\hline & Dendrophora albo-badia (Sch.: Fr.) Chamuris & & $\mathrm{RE}, \mathrm{CE}$ \\
\hline & Dentipellis fragilis (Pers.: Fr.) Donk & & $\mathrm{RE}, \mathrm{CE}, \mathrm{CO}$ \\
\hline & Hyphoderma guttuliferum (Karst.) Donk & & $\mathrm{RE}, \mathrm{CE}$ \\
\hline & Peniophorasp & & $\mathrm{RE}, \mathrm{CE}$ \\
\hline & Phanerochaete sordida (Karst.) Erikss. \& Ryy. & & $\mathrm{RE}, \mathrm{CE}, \mathrm{CO}$ \\
\hline & Porostereum amethysteum Hjortst. \& Ryv. & & $R E, C E, C O$ \\
\hline & Stereum strigosa-zonatum (Sch.) G.H. Cunn. & & $R E, C E$, \\
\hline Dacryomycetaceae & Dacryopinax spathularia (Sch.) Martin & & $\mathrm{RE}, \mathrm{CE}$ \\
\hline Lachnocladiaceae & Vararia investiens (Schw.) Karst. & & $\mathrm{RE}, \mathrm{CE}, \mathrm{CO}$ \\
\hline \multirow[t]{2}{*}{ Lentinaceae } & Panus crinitus (L.: Fr.) Sing. & & $\mathrm{RE}, \mathrm{CE} ; \mathrm{MA}, \mathrm{CE}, \mathrm{CO} M \mathrm{MACE}, \mathrm{CO}$ \\
\hline & Panus nicotianus Berk & & $\mathrm{RE}, \mathrm{CE} ; \mathrm{MA}, \mathrm{CE}, \mathrm{CO}$ \\
\hline Orbiliaceae & Orbilia auricolor (Blox.) Sacc. & & $\mathrm{RE}, \mathrm{CE}$ \\
\hline \multirow[t]{13}{*}{ Polyporaceae } & Ceriporia mellea (Berk. \& Br.) Ryv. & & $\mathrm{RE}, \mathrm{CE}$ \\
\hline & Junghuhnia crustacea (Jungh.) Ryv. & & $\mathrm{RE}, \mathrm{CE}$ \\
\hline & Hymenogramme javanensis Mont. \& Berk. & RE & \\
\hline & Lenzites striata (Swart.: Fr.) Fr. & & MA, CE, CO \\
\hline & Lenzites sp & & $M A, C E, C O$ \\
\hline & Oligoporus hibernicus (Berk. \& Br.) Gillbn. \& Ry. & & $\mathrm{RE}, \mathrm{CE}$ \\
\hline & Oligoporus placentus (Fr.) Gilbn. \& Ryv. & & $\mathrm{RE}, \mathrm{CE}$ \\
\hline & Pycnoporus sanguineus (L.: Fr.) Murr. & & $\mathrm{RE}, \mathrm{CE} ; \mathrm{MA}, \mathrm{CE}, \mathrm{CO}$ \\
\hline & Schizopora paradoxa (Fr.) Donk & & $R E, C E, C O$ \\
\hline & Skeletocutis sp & & $\mathrm{RE}, \mathrm{CE}$ \\
\hline & Trametes cf. cotonea (Pat. \& Har.) Ryv. & & $\mathrm{RE}, \mathrm{CE}$ \\
\hline & Trametes of. pubescens (Schum.: Fr.) Pil. & & $\mathrm{RE}, \mathrm{CE}$ \\
\hline & Trametes villosa ( $\mathrm{Fr}$.) Kreis. & & $\mathrm{RE}, \mathrm{CE}$ \\
\hline \multirow[t]{2}{*}{ Schyzophyllaceae } & Schizophyllum commune Fr. & & $\mathrm{RE}, \mathrm{CE} ; \mathrm{MA}, \mathrm{CE}$ \\
\hline & Schizophyllum fasciatum Pat. & & $\mathrm{RE}, \mathrm{CE} ; \mathrm{MA}, \mathrm{CE}$ \\
\hline \multirow[t]{5}{*}{ Xylariaceae } & Daldinia concentrica (Bolton. :Fr.) Ces. \& de Not. & & $\mathrm{RE}, \mathrm{CE}$ \\
\hline & Hypoxylon rubiginosum (Pers.: $\mathrm{Fr}$.) $\mathrm{Fr}$ & RE & \\
\hline & Phaeiosoria sp & & $\mathrm{RE}, \mathrm{CE}$ \\
\hline & Rhytidhysteron rufulum (Spreng.) Petr. & & $\mathrm{RE}, \mathrm{CE}$ \\
\hline & Nodulisporium gregorium (B. \& C.) Meyer & & $\mathrm{RE}, \mathrm{CE}$ \\
\hline
\end{tabular}

$\mathrm{RE}=$ revestimento $\mathrm{MA}=$ madeira $\mathrm{ME}=$ medula $\quad \mathrm{CE}=$ posição convexa $\quad \mathrm{CO}=$ posição côncava 
em outro trabalho.

\section{Durabilidade natural da madeira de $B$. gasipaes em condições de laboratório}

A madeira de ambas as variedades da pupunheira apresentou média inferior ou igual a $4,12 \%$ de perda de massa e a madeira de $S$. amara (testemunha) apresentou perda de massa de $30,71 \%$ à $P$. fumosus, $34,35 \%$ à $P$. sanguineus e de $61,94 \%$ à L. trabea (Tab. 7). Com base nestes dados e de acordo com a classificação da resistência da madeira expostas à condições de laboratório (Tabela 3), a madeira de $B$. gasipaes pode ser classificada como altamente resistente aos fungos testados, assim como a madeira de $S$. amara pode ser classificada como não resistente à $L$. striata e moderadamente resistente à $P$. sanguineus e $P$. fumosus.

Os resultados obtidos para a durabilidade de $B$. gasipaes podem ser explicados em virtude que durante a autoclavagem a $120^{\circ} \mathrm{C}$, aparentemente as amostras liberaram uma substância oleosa, a qual, no bioensaio, promoveu a formação de uma película micelial na interface entre o fungo e a madeira, o que poderia ter funcionado como um mecanismo de proteção ou como uma substância antifúngica, inibindo o desenvolvimento do fungo na madeira.

Esta alteração pode estar associada a influencia da alta temperatura na composição química que pode ter favorecido a oxidação em algum composto com propriedades antifúngicas, considerando que extratos obtidos da madeira e do revestimento de $B$. gasipaes avaliados frente às mesmas espécies de fungos, não apresentaram atividades antifúngicas (Teixeira et al., 1997). As divergências nestes dados sugerem que no bioensaio da durabilidade de madeira da pupunheira, a esterilização das amostras deve ser realizada por outros métodos alternativos à alta temperatura. Por outro lado, a alta densidade da madeira da pupunha de $1,07 \mathrm{~g} / \mathrm{cm}^{3}$ (Lobato et. al., 1999) também pode ter contribuído para a durabilidade natural aos fungos testados.

Em comparação com outras palmeiras, a resistência natural apresentada pela madeira de $B$. gasipaes difere da madeira de Phoenix canariensis, que demostrou ser altamente suscetível aos fungos apodrecedores em testes em agar/

Tabela 7. Média da perda de massa (\%) e classe de resistência da madeira da pupunheira com e sem espinhos e de marupá em contato com os fungos apodrecedores.

\begin{tabular}{|c|c|c|c|c|c|c|}
\hline \multirow[b]{3}{*}{ Madeira } & \multicolumn{6}{|c|}{ Fungos apodrecedores } \\
\hline & \multicolumn{2}{|c|}{ Lenzites trabea } & \multicolumn{2}{|c|}{ Pycnoporus sanguineus } & \multicolumn{2}{|c|}{ Polyporus fumosus } \\
\hline & $\begin{array}{l}\text { Perda de } \\
\text { massa(\%) }\end{array}$ & $\begin{array}{l}\text { Classe de } \\
\text { resistência }\end{array}$ & $\begin{array}{l}\text { Perda de } \\
\text { massa(\%) }\end{array}$ & $\begin{array}{l}\text { Classe de } \\
\text { resistência }\end{array}$ & $\begin{array}{l}\text { Perda de } \\
\text { massa(\%) }\end{array}$ & $\begin{array}{l}\text { Classe de } \\
\text { resistência }\end{array}$ \\
\hline Pupunheira com espinhos & 2,9 & $A R$ & 1,83 & $A R$ & 4,12 & AR \\
\hline Pupunheira sem espinhos & 1,19 & AR & 1,19 & AR & 2,5 & AR \\
\hline Marupá & 61,94 & NR & 34,35 & MR & 30,71 & MR \\
\hline
\end{tabular}


bloco (Adaskaveg et al.; 1990).

\section{Conclusão}

A madeira de $B$. gasipaes, exposta em ambiente florestal, demonstra ser moderadamente resistente aos fungos, enquanto que a madeira exposta ao ambiente urbano apresenta alta susceptibilidade a diferentes espécies de fungos apodrecedores. Dentre os fungos associados a biodegradação das amostras expostas ao ambiente urbano, destacam-.se, L. striata, Lenzites sp, $P$. amethysteum e $S$. strigoso-zonatum.

Em ensaios de laboratório, a madeira é considerada altamente resistente aos fungos $P$. fumosus, $P$. sanguineus e $L$. trabea.

Do ponto de vista da aplicabilidade econômica da madeira de B. gasipaes (Pupunha), os dados obtidos da durabilidade da madeira em ambiente florestal e urbano sugerem que a madeira não é recomendada para uso em ambiente externo devido à alta suscetibilidade aos fungos xilófagos. Neste caso, a madeira deve ser preservada com fungicidas.

\section{Agradecimentos}

Ao Dr. Jacques Boidin da França pela classificação dos fungos apodrecedores de madeira e ao Dr. Basilio Frasco Vianez do INPA pelas sugestões ao texto do trabalho.

\section{Bibliografia citada}

Adaskaveg, J.E.; Blanchette, R.; Gilbertson, R. 1990. Decay of date palm wood by whiterot and brow-rot fungi. Can. J. Bot., 69:
615-629.

American Society For Testing and Material. 1981. Standard methods of accelerated laboratory test of natural decay resistance of woods. In: Annual Book of ASTM Standards, Wood and Adhesives. Part. 22. Philadelphia, USA. p. 639-645.

Araújo, J.C. 1991. Aspectos técnicos da implantação da cultura da pupunheira para produçāo de palmito. In: A Pupunheira e suas potencialidades econômicas. Secretaria de Produçāo Rural (SEPROR), Manaus, 37p.

Cavalcante, M.S.; Montagna, R.G.; Lopes, G.A.C.; Mucci, F. 1982. Durabilidade de madeira em contato com o solo. II. Silvicultura em São Paulo. Rev Inst. Forestal, vol.16. Pte 2, p1383-88p.

Cavalcanti, M.A.Q. 1983. Basidiomicetos poliporóides destruidores de madeira em serrarias do Recife. Rev. Pernamb. Tec. 3 (3):83-87.

INPA/CPPF. 1991. Catálogo de madeiras da Amazônia: Características tecnológicas; Área da Hidrelétrica de Balbina. CPPF/ INPA, Manaus, 163p.

Falesi, I.C. 1971. Solos do Distrito Agropecuário da SUFRAMA (Trecho: Km 30-Km 79, Rod. BR 174). Série Solos, (Supl. 1). IPEAM, Manaus, 99p.

Gomes, J.B.M. 1993. Crescimento e produção da Pupunha (Bactris gasipaes Kunth) consorciada com mandioca (Maniato suculenta Carnaz), Uruçu (Bisa orellana L.), banana pacovão (Musa paradíaca) e abacaxi (Ananas comosus.) $L$. Dissertação de mestrado. Instituto Nacional da Amazônia/Fundação Universidade do Amazonas. Manaus, Amazonas. 91p.

Jesus, M. A. 1995. Contribution to the knowledge of wood-rotting fungi in Brazil. I. Occurrence and distribution of fungi on different substrates from the Manaus region, Amazonas State. Ann. Meeting. The Int. Res. Group on Wood Pres. (Doc. IRG/ WP95-10096). 19p.

Hjortstam, K.; Ryvarden, L. 1990. Lopharia and porostereum (Corticiaceae). Synopsis 
Fungorum 4, Fungiflora, Oslo. Norway. $68 \mathrm{p}$.

Lobato, R.H.; Rocha, J.S.; Bessa, T.M. 1999. Aproveitamento do estipe da pupunha (Bactris gasipaes Kunth) para móveis. Anais da VII Jornada de Iniciação Cientifica do PIBIC/INPA/CNPq. Manaus, p. 219-222.

Lorenzo, H.; Souza, H.M.; Medeiros-Costa, J.T.; Cerqueira. L.S.C. 1996. Palmeiras no Brasil nativas e exóticas. Editora. Plantarum. Nova Odessa, São Paulo, 303p.

Marchiori, J.N.C. 1995. Elementos de Dendrologia. Editora da UFSM, Santa Maria, 163p.

Milano, S.; Chimelo, J.; Bononi, V. 1985. Decay fungi collected in Brazil from sleepers during the air seasoning period. Ann. Meeting. The Int. Res. Group on Wood Pres. (Doc. IRG/85). 19p.

Mora Urpí, J. 1984. El pejibaye (Bactris gasipaes H.B.K.): origen, biologíafloral y manejo agronómico. In: Palmeras poco utilizadas de América Tropical. Food and Agriculture Organization (FAO)/Centro Agronómico Tropical de Investigación y Ensenanza (CATIE), Turrialba,Costa Rica, p.118-160.

Ortós, H.1975. Forest fungi against the background of environment. U.S Depart. Agric. and the Nat. Scie. Foundation, Washington D.C.USA. $187 \mathrm{p}$.

Overholts, L.O. 1977. The polyporaceae of the Unites States, Alaska and Canada. Univ. Michigan Press. Ann Arbor, Michigan, USA. 466p.

Patiño, V.M. 1963. Plantas cultivadas y animales domésticos en América Equinoccial. I. Frutales. Imprenta Departamental, Cali, Colombia. 547p.

Ryvarden, L.; Johansen, I. 1980. A preliminar polypore flora of East Africa. Fungiflora, Oslo. Norway. 636p.

Serpa, F.G. 1982. Durabilidade natural de madeiras do nordeste em campo de apodrecimento. Rev. Pernam. Tec., (1):47-54.

Sutter, H. P. 1978. A new technique for screening fungicides for wood preservatives.
(Doc. ISSN 0020-6164) p. 95-99.

Teixeira, L. M.; Jesus, M. A.; Varejão, M.J.C. 1997. Avaliação da toxicidade de extrativos da Pupunha (Bactris gasipaes) Kunth à fungos xilófagos. Anais da VIJ. Cient. PIBIC/INPA/CNPq. Manaus, p.119-120.

Vieira, L.S.; Santos, P.C.T.C. 1987. Amazônia: seus solos e outros recursos naturais. Editora Agronômica Ceres. São Paulo, 416p.

Aceito para publicação em 05/08/2002. 
